\title{
OPTIMUM POSTSTALL TURNING AND SUPERSONIC TURNING
}

\author{
Nguyen X. Vinh ${ }^{*}$ and Yih- Feng Tzeng ${ }^{\dagger}$ \\ University of Michigan, Ann Arbor, Michigan 48109
}

\begin{abstract}
In this paper we consider the optimum maneuvers for the fastest turning and the turning with the smallest radius in nearly horizontal flight. It is shown that by retaining the thrust component along the direction of the lift force, the optimum angle-of-attack for the maneuvers is beyond the usual stall angle-of-attack where the lift coefficient reaches its maximum before decreasing drastically. For very high thrust-to-weight ratio, optimum turn is performed at low speed and at the maximum angle-of-attack allowed. For fastest turn subject to prescribed end-points Mach numbers, the optimum technique in bank and thrustcontrol depends on the limitation on the load factor, aerodynamic and propulsive capabilities. Explicit control laws for the bank angle and for the thrust are derived and numerical examples for optimum turning are presented.
\end{abstract}

\section{Introduction}

For high-performance fighter aircraft, the thrust-toweight ratio is high, especially at low altitude, and the turning performance depends heavily on the aerodynamic and the physiological-structural constraints and the discussion of the optimum maneuvers is rather subtle.

In this paper we consider the optimum maneuvers for the fastest turning and the turning with the smallest radius in nearly horizontal flight. In the analysis we have removed the simplifying assumptions used in the standard texts ${ }^{1,2}$ to include all the non-linear forces in the dynamical system. In this respect, the following steps are taken :

(a) We retain the thrust component $T \sin \alpha$ in the direction of the lift force.

(b) We allow the angle-of-attack to get beyond the stall angle-of-attack at which the lift coefficient passes through a maximum and starts to decrease drastically. Then, instead of using the standard parabolic drag polar, the non-linear variations of the lift coefficient and the drag coefficient up to a very high angle-ofattack $\alpha_{\max }=70^{\circ}$ are considered. We have

\footnotetext{
* Professor, Department of Aerospace Engineering. Member AIAA

† Graduate Student, Department of Aerospace Engineering.

Copyright @ 1993 by the American Institute of Aeronautics and Astronautics, Inc. All rights reserved.
}

$$
\begin{aligned}
& C_{L}=C_{L}(M, \alpha) \\
& C_{D}=C_{D}(M, \alpha)
\end{aligned}
$$

with enough data points on the Mach number $\mathrm{M}$ and the angle-of-attack $\mathbf{Q}$ for a smooth spline approximation.

(c) The maximum thrust, generally used for fastest turning, strongly depends on the altitude and the Mach number. Then we represent

$\mathrm{T}_{\max }=\mathrm{T}_{\max }(h, M)$

with enough data points to cover the domain of interest. Since the optimum maneuver depends on this maximum thrust magnitude, we consider two jet engines with respectively high and moderate thrust-toweight ratio in the analysis.

(d) We consider both steady turn and non-steady tum. For non-steady turn, optimal control theory is the tool for the analysis.

\section{Equations of Motion}

For nearly horizontal flight, the flight path angle is negligibly small. This is to say that, for all practical purposes, we can consider the flight at constant altitude. The equations for the motion of the center of mass are

$$
\begin{aligned}
& \frac{d x}{d t}=V \cos \psi \\
& \frac{d y}{d t}=V \sin \psi \\
& d V=g(T \cos \alpha-D) \\
& d t w \\
& \frac{d \psi}{d t}=\frac{g}{V W}(T \sin \alpha+L) \sin +
\end{aligned}
$$

where $\mathrm{x}$ and $\mathrm{y}$ are the Cartesian coordinates in the horizontal plane, $\mathrm{V}$ is the speed and $\psi$ is the heading measured from the $\mathbf{x}$-axis. The angle-of-attack is measured from the thrust line and the bank angle $\phi$ is defined as the angle which measures the rotation of the lift force out of the vertical 
plane (Fig. 1). For the short duration of the flight, the weight $\mathrm{W}$ is practically constant. drag force

We use the standard assumption for the lift and the

$$
\begin{aligned}
& L=q S C_{L} \\
& D=q S C_{D}
\end{aligned}
$$

where $q=\frac{1}{2} \rho V^{2}$ is the dynamic pressure, $S$ is the reference area and, at any flight altitude, the atmospheric mass density $\rho$ is a well specified constant. We also have the speed of sound at the flight altitude. Thus, at any flight altitude, the aerodynamic characteristics and the maximum thrust produced depend on the current flight speed, through the Mach number.

For a coordinated turn at constant altitude, the vertical component of the combined lift and propulsive force is used to balance the weight. Hence, we have the constraining relation

$$
(\mathrm{T} \sin a+\mathrm{L}) \cos \phi=\mathrm{W}
$$

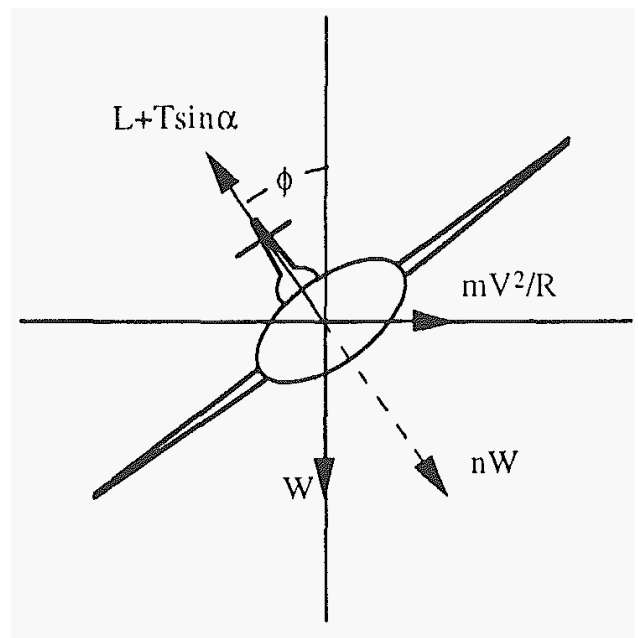

Fig. 1 Force diagram in a coordinated turn

The turning is controlled by the thrust magnitude $\mathrm{T}$, the angle-of-attack $a$ and the bank angle $\phi$. Because of the constraining relation (5), only two components of the control, out of the three, are independent.

$$
\begin{aligned}
& \text { If we rewrite Eq. (5) } \\
& \mathrm{T} \sin \alpha+\mathrm{L}=\mathrm{n} W
\end{aligned}
$$

we can use this relation as the definition of the load factor n. There exists a physiological-structural constraint on this load factor which is easily encountered at low altitude and for turn at high bank angle. By comparing the last two equations, we see that

$$
n=\frac{1}{\cos \phi}
$$

Therefore, if the load factor constraint is set at $n_{s}=6$, the bank angle is limited at $\phi_{\max }=80.41^{\circ}$.

The two parameters of interest in turning flight are the turning rate $\psi$ which is given by Eq. (3d) and the turning radius $\mathrm{R}$. For the general case of non-steady turn, the turning radius of curvature is given by

$$
\frac{1}{R}=\frac{g}{V^{2} W}(T \sin a+L) \sin \phi
$$

In point performance, at any instant of time, we want to use the control, subject to physical constraints to have the maximum turning rate $\dot{\psi}_{\max }$ or the minimum turning radius of curvature $R_{\min }$. If those optimum quantities can be maintained constant, the turning is steady. If the thrust is not sufficiently high, the optimum performance is time varying.

In integral performance we may consider the problem of the fastest turn which consists of bringing the vehicle from an initial Mach number $\mathbf{M}_{0}$, from an initial direction $\psi_{0}=0^{\circ}$, to a final Mach number $M_{\mathrm{f}}$ with a specified final heading $\psi_{\mathrm{f}}$ in minimum time, The search for the optimum control requires the variational analysis.

\section{Point Performance}

By using Eq. (6) for the load factor, we rewrite Eq. (3d) for the turning rate

$$
\dot{\psi}=\frac{\mathrm{g}}{\mathrm{a} M} \sqrt{\mathrm{n}^{2}-1}
$$

where $\mathrm{a}$ is the speed of sound at the flight altitude. Therefore, at any current Mach number the highest instantaneous turning rate is obtained by using the maximum load factor. From Eq. (6), we maximize

$$
\mathrm{n}=\frac{\mathrm{T}}{\mathrm{W}} \sin a+\underset{\mathrm{W}}{\mathrm{t}} \leq \mathrm{n}_{\mathrm{s}}
$$

We write explicitly the expression for $n$ as function of the Mach number and the angle-of-attack

$$
n=\eta \frac{T_{\max }(M, h)}{W} \sin \alpha+\frac{q S}{W} C_{L}(M, \alpha)
$$


where $\eta \mathbf{E}[0,1]$ is the thrust control. It is obvious that for any $M$ and $\alpha$, we should use $\eta=1$ for maximum of $n$. At any current Mach number, we consider the equation

$$
\frac{\partial \mathrm{n}}{\partial \alpha}=\eta \frac{\mathrm{T}_{\max }(\mathrm{M}, \mathrm{h})}{\mathrm{W}} \cos \alpha+\frac{\mathrm{q} S}{\mathrm{~W}} \frac{\partial \mathrm{C}_{\mathrm{L}}}{\partial \alpha}=0
$$

For this equation to have a solution, it is necessary that $\partial \mathrm{C}_{\mathrm{L}} / \partial \alpha<0$. Since $\partial \mathrm{C}_{\mathrm{J}} / \partial \alpha=0$ at the stall angle-of attack, the maximum load factor for fastest instantaneous turning rate occur's beyond the stall angle-of-attack.

By rewriting Eq. (8) for the turning radius as

$$
R=\frac{a^{2} M^{2}}{g \sqrt{n^{2}-1}}
$$

we can see that the optimum angle-of-attack, solution of Eq. (12) also provides the minimum instantaneous turning radius. We should notice that if the corresponding value of the instantaneous maximum $n=n_{\max }(M)$ exceeds the value $n_{s}$ which is arbitrarily imposed, then the fastest turn is conducted at $n=n_{s}$.

We now search for the optimum value of the Mach number for a maximum of $\mathrm{n}$. It is obtained, at any value for $\alpha$, by solving the equation

$$
\frac{\partial \mathrm{n}}{\partial \mathrm{M}}-\underset{\mathrm{W}}{\eta} \partial \mathrm{T} \mathrm{T}_{\max } \sin \alpha+\frac{\mathrm{qS}}{\mathrm{W}}\left(\frac{\partial \mathrm{C}_{\mathrm{L}}}{\partial \mathrm{M}}+\frac{2}{\mathrm{M}} \mathrm{CL}\right)=0
$$

For realistic values of $\mathrm{T}_{\max } / \mathrm{W}$, the optimum angle-of-attack for maximum load factor is slightly beyond the stall angleof-attack. Therefore, an approximate solution to Eq. (14) is obtained with $\alpha=\alpha_{\text {stall }}$ and $C_{L}=C_{\mathrm{Lmax}}(M)$. Since in general $\mathrm{T}_{\max }$ increases with the Mach number, the solution occurs at high subsonic speed when $\mathrm{C}_{\mathrm{Lmax}}$ has a sharp decrease.

If we remove the constraint $n_{s}$, then using the solution (12) for $\alpha$ in Eq.(11), we should obtain the maximized load factor as function of the Mach number, that is

$$
\mathrm{n}=\mathrm{n}_{\max }(\mathrm{M})
$$

A typical plot of this function is shown in Fig. 2. Then, it appears that, there exists a Mach number $\mathrm{M}_{*}$ for maximum $\mathrm{n}$, the solution of which is obtained by solving the two cquations (12) and (14) for $\alpha_{*}$ and $M_{*}$. By using Eq. (15) in Eq. (9) for the turning rate and Eq. (13) for the turning radius, it is now seen that they are functions of the Mach number. It has been shown in Ref. 2 that there exist an optimum value $\mathrm{M}=\mathrm{M}_{\mathrm{opt}}(\dot{\psi})$ which maximizes the turning rate and an optimum value $\mathrm{M}=\mathrm{M}_{\text {opt }}(\mathrm{R})$ which minimizes the furning radius and that

$$
\mathrm{M} *>\mathrm{M}_{\mathrm{opt}}(\dot{\psi})>\mathrm{M}_{\mathrm{opt}}(\mathrm{R})
$$

With the new definition of the load factor, the same proof can now be applied to the present case where the thrust component $T \sin \alpha$ is included in the lift direction.

In the case where $\mathbf{n}_{\max }>n_{s}$, optimum turn has to be conducted at $n=n_{s}$. Then from Eqs. (9) and (13) the fastest turn and the turn with the smallest radius is conducted with the smallest possible Mach number. With the component Tsin $\alpha$ included, this so-called coiner Mach number, and the associated angle-of-attack, are obtained by solving Eq. (11) with $n=n_{s}$ and Eq. (12).

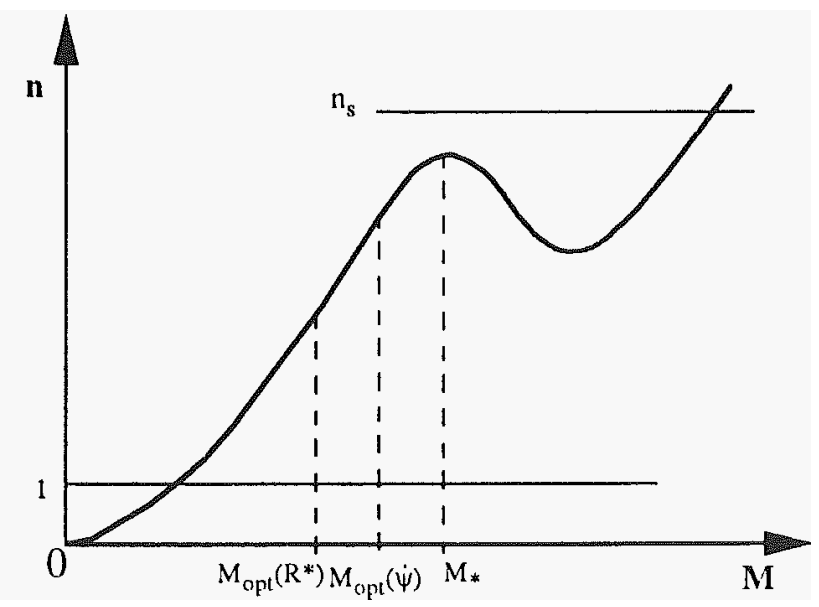

Fig. 2 Maximum load factor as function of the Mach number at any flight altitude

\section{$\underline{\text { Steady Turn }}$}

In the previous section, we have displayed the existence of the absolute maximum turning rate and the absolute minimum radius of curvature. Each of them occurs at any given flight altitude at a certain poststall angle-of-attack and an optimum Mach number which can be computed once the physical characteristics of the aircraft are given. In general, we are dealing with high angle-of-attack, high subsonic speed, and high load factor. Hence the resulting drag force is high and this will require a high thrust-to-weight ratio to maintain the optimum turning condition. More specifically, by writing the condition $\mathrm{dV} / \mathrm{d} t=0$, we have

$$
\eta \mathrm{T}_{\max } \cos \alpha=q S \mathrm{C}_{\mathrm{D}}(\mathrm{M}, \alpha)
$$

and for high drag, this condition may not hold. For steady turn, the controls $\eta, \alpha$, and $n$ must be such that the two equations (11) and (17) are constantly satisfied. Assume that we have selected a throttle setting value $\eta$. Then for 
any 'angle-of-attack $\alpha$ used for tuning, the constant speed is obtained from Eq. (17), and the load factor $n$ is deduced from Eq. (11), with the corresponding bank angle from Eq. (7). The turning rate is given by Eq. (9) and the constant turning radius is given by Eq. (13).

By using Eq. (11)in Eq. (9) we obtain

$$
\dot{\psi}=\frac{\mathrm{g}}{\mathrm{a} M \mathrm{~W}} \sqrt{\left(\mathrm{q} S \mathrm{C}_{\mathrm{L}}+\eta \mathrm{T}_{\max } \sin \alpha\right)^{2}-\mathrm{W}^{2}}
$$

With $C_{L}=C_{L}(M, \alpha), q=\frac{1}{2} \rho a^{2} M^{2}, T_{\max }=T_{\max }(M, h)$ for any $\eta$, this is a function of $\alpha$ and $\mathrm{M}$, subject to the constraint (17). We can routinely search for the maximum turning rate.

as

Similarly, we can write the constant turning radius

$$
\mathrm{R}=\frac{\mathrm{aM}}{\dot{\psi}}
$$

and minimize this quantity subject to the same constraint (17)

\section{Numerical Application}

We first consider the case of steady turn with a prescribed thrust at its maximum value. Then, by using Eq. (17) for qS in Eq. (11), we have

$$
\mathrm{n}=\tau\left(\frac{\mathrm{C}_{\mathrm{L}}}{\mathrm{C}_{\mathrm{D}}} \cos \alpha+\sin \alpha\right)
$$

where

$$
\tau=\frac{T}{W}
$$

is the thrust-to-weight ratio.

To display the poststall turning which occurs at low speed, we assume that $\mathrm{z}, \mathrm{C}_{\mathrm{L}}$ and $\mathrm{C}_{\mathrm{D}}$ are independent of Mach number. Then $\mathrm{n}$ is a function of the angle-of-attack. We can also express the dimensionless turning rate and the dimensionless turning radius at any flight altitude as

$$
\frac{\dot{\Psi}}{\mathrm{g}} \sqrt{\frac{2 \mathrm{~W} \tau}{\rho \mathrm{S}}}=\overline{\dot{\psi}}=\sqrt{\frac{\mathrm{C}_{\mathrm{D}}\left(\mathrm{n}^{2}-1\right)}{\cos \alpha}}
$$

and

$$
\frac{\mathrm{Rg} \rho S}{2 \mathrm{~W} \tau}=\overline{\mathrm{R}}=\frac{\cos \alpha}{\mathrm{C}_{\mathrm{D}} \sqrt{\left(\mathrm{n}^{2}-1\right)}}
$$

By using $\tau$ as a varying parameter, we have plotted the load factor, the dimensionless turning rate and the dimensionless turning radius using the aerodynamic characteristic $C_{L}(\alpha)$ and $C_{D}(\alpha)$ of the aircraft in Ref. 3 as functions of the angle-of-attack in Figs. 3, 4 and 5.

Let $E=C_{L} / C_{D}$ be the lift-to-drag ratio. We can prove the following assessments.

Consider the equation

$$
\frac{d n}{d a}=\tau\left[\left(\frac{d E}{d a}+1\right) \cos \alpha-E \sin \alpha\right]=0
$$

When $\alpha \approx 0, \operatorname{dn} / d \alpha>0$. For a typical drag polar, at high angle-of-attack, $\cos \alpha$ is small and $\sin \alpha$ is large, while $\mathrm{dE} / \mathrm{d} \alpha$ becomes negative. Then $\mathrm{dn} / \mathrm{d} \alpha<0$. Therefore, $\mathrm{n}$ passes through a maximum at a value $\alpha_{*}$ independent of $\tau$. If small angle approximation is used in Eq. (20), we can see that $\alpha_{*}$ is slightly larger than the optimum value for maximum lift-to-drag ratio. Furthermore, it is seen in the same equation that, for very large value of $\alpha, \mathrm{n}$ tends asymptotically to $\tau$.

From Eq. (22), at any altitude and thrust-to-weight ratio, the angle-of-attack which maximizes the turning rate is the one giving the maximum of the function

$$
\mathrm{F}=\frac{\operatorname{CD}\left(\mathrm{n}^{2}-1\right)}{\cos \mathrm{a}}
$$

we consider the derivative

$$
\frac{d E}{d \alpha}=\frac{C_{D}}{\cos \alpha}\left[2 n \frac{d n}{d \alpha}+\left(n^{2}-1\right)\left(\tan \alpha+\frac{1}{C_{D}} \frac{d C_{D}}{d \alpha}\right)\right]
$$

Notice that for turning $n>1$ and we always have $\mathrm{dC}_{\mathrm{D}} / \mathrm{d} \alpha>0$. Therefore, if a relative maximum of the tuining rate exists, it must occur beyond the angle-of-attack for maximum load factor since it would require $\mathrm{dn} / \mathrm{d} \alpha<0$ for $\mathrm{dF} / \mathrm{d} \alpha$ to vanish.

From Eq.(23), we see that to minimize the turning radius, we maximize the function

$$
G=\frac{C_{D}^{2}\left(n^{2}-1\right)}{\cos ^{2} \alpha}=\frac{C_{D}}{\cos \alpha} F
$$

Then, we consider the derivative

$$
\frac{\mathrm{dG}}{\mathrm{d} \alpha}=\frac{\mathrm{C}_{\mathrm{D}}}{\cos \alpha}\left[\frac{\mathrm{dF}}{\mathrm{d} \alpha}+\mathrm{F}\left(\tan \alpha+\frac{1}{\mathrm{C}_{\mathrm{D}}} \frac{\mathrm{d} \mathrm{C}_{\mathrm{D}}}{\mathrm{d} \alpha}\right)\right]
$$

For this derivative to vanish, it requires that $d F / d \alpha<0$. The angle-of-attack for a relative minimum of the turning radius 
is beyond the angle-of-attack for maximum turning rate. In summary, we have

$$
\alpha *<\alpha_{\text {opt }}(\dot{\psi})<\alpha_{\text {opt }}(\mathrm{R})
$$

From Fig. 4, we see that although there exists a value $\alpha_{\text {opt }}(\dot{\psi})$ which depends on $\tau$ for a relative maximum of the turning rate, for very high values of $\tau$, the highest poststall turning rate may occur at the highest angle-of-attack $\alpha_{\max }=70^{\circ}$. This is also true for the case of minimum turning radius as can be clearly seen in Fig. 5 .

To prepare for next section in which we use optimum control theory to obtain the fastest turn with boundary conditions for the current generation of fighter aircraft with constraints on load factor and maximum thrust output we search for the absolute maximum turning rate. Quite frequently, in problems of practical interest, the endpoints Mach numbers are high and as such the angles-ofattack involved are small, Therefore in the computation, we neglect the component Tsin $\alpha$ and as a consequence the load factor is simply the lift-to-weight ratio. We use the physical characteristics of the example aircraft in Ref. 4, with some specifications reported in the Appendix. The atmosphere used for $\rho(h)$ and $a(h)$ is the Standard atmosphere.

Since the physical constraints on the problem are imposed on the load factor and the maximum thrust produced, we consider the plot on the $(M, n)$ space. By using the relation

$$
\mathrm{a}^{2}=\frac{\mathrm{k} \mathrm{p}}{\rho}
$$

where $p$ is the pressure and $\mathrm{k}$ is the ratio of the specific heats with $k=1.4$ for air, we can write Eq. (11) with the term Tsin $\alpha$ neglected as

$$
\mathrm{n}=\left(\frac{\mathrm{kpS}}{2 \mathrm{w}}\right) \mathrm{M}^{2} \mathrm{C}_{\mathrm{L}}(\alpha, \mathrm{M})
$$

By specifying two flight altitudes at $5 \mathrm{kft}$ and 14 $\mathrm{kft}$ with the corresponding values for p, we plot in Figs. 6 and 7 the maximum of $n$ versus the Mach number by using $\alpha=\alpha_{\text {stall, }}$, and hence $C_{L}=C_{\text {Lmax }}(M)$ in Ey. (31). We first refer to Fig. 6 for the discussion. Concerning pure aerodynamic limit with $\mathrm{C}_{\mathrm{L}}=\mathrm{C}_{\mathrm{Lmax}}$, the load factor passes through a maximum $n_{\max }=8.405$ at $M=0.725$, and then decreases and passes through a minimum and finally increases at high Mach number. This is represented by the curve 1 in the $(\mathrm{M}, \mathrm{n})$ space. As has been discussed in the section Point Performance, with the maximized $n$ obtained as function of $\mathrm{M}$, we can plot the turning rate as given in Eq. (9) as a function of $M$ and it is shown in the curve 5 in this figure. But if we enforce the structural limit at $n_{s}=6$, the $(M, n)$ space is restricted to be below this line $\mathrm{CF}$. The maximized turning rate is computed with $n=6$ whenever $\mathrm{n} \max (\mathrm{M})>\mathrm{n}_{\mathrm{s}}$, and the turning rate is reduced with this constraint to the curve 6 , with a maximum at the corner Mach number, at point $\mathrm{C}$ on the $(\mathrm{M}, \mathrm{n})$ space.

In summary, at any Mach number, for maximum turning rate, we use the maximum value of $n$ on the boundary, with the overall absolute maximum turning rate at the point $\mathrm{C}$. Ideally, we should quickly bring the speed to this optimum Mach number and use the thrust to maintain this speed for the fastest rate. We now, introduce the maximum thrust for steady tum at the selected load factor. For this purpose, we use a parabolic drag polar of the form

$$
\mathrm{C}_{\mathrm{D}}=\mathrm{C}_{\mathrm{D} 0}(\mathrm{M})+\mathrm{K}(\mathrm{M}) \mathrm{C}_{\mathrm{L}}^{2}
$$

to plot the equation

$$
\mathrm{T}_{\max }(\mathrm{M}, \mathrm{h})=\mathrm{D}\left[\mathrm{M}, \mathrm{h}, \mathrm{C}_{\mathrm{D}}\left(\mathrm{C}_{\mathrm{L}}\right)\right]
$$

By using $C_{D}$ from Eq. (32) and then $C_{L}$ from Eq. (31), it is seen that Eq. (33) represents a curve in the (M,n) space. This theory is also valid for any arbitrary drag polar. We have used two jet engines which lead to the curves 3 and 4 in the figure. Steady turn can be maintained below the curves where $\mathrm{T}_{\max }>\mathrm{D}$ and hence at an intermediate value of the thrust. As seen in this figure, both the engine thrusts are riot sufficiently high to maintain the peak tuming rate at the point $\mathrm{C}$ and the turning technique is to intercalate the maximum thrust with the null thrust at a varying and slower rate as to satisfy the end-conditions. This will be discussed in the next section.

In Fig. 7, we have use the same example aircraft with the same two engines but with the analysis at a higher altitude $h=14 \mathrm{kft}$.

Again, the curve 1 is the plot of the maximum load factor as a function of the Mach number and it passes through a relative maximum at the point $\mathrm{C}_{1}$. With this function $\mathrm{n}=\mathrm{n}_{\max }(M)$, we plot the tuiiiing rate $\dot{\psi}$ as function of the Mach number in curve 5, and, as the theory has predicted, there exists a local maximum turning rate at point $S_{1}$ at the optimum Mach number $M_{\text {opt }}(\dot{\psi})$ less than the value $M_{*}$ at point $C_{1}$. If we enforce the constraint $n \leq 6$ the usable domain for $\mathrm{M}$ and $\mathrm{n}$, which we refer to as the domain of maneuverability, is restricted to the space bounded by both the aerodynamic limit and the structural limit. Then there appears another local peak turning at point $S_{2}$ for the corner Mach number from point $C_{2}$, intersection of the aerodynamic limit and the constraint $n=n_{s}$. In summary, at this altitude with the constraint $n \leq n_{s}$ enforced, at any current Mach number regardless of the engine thrust, by taking the instantaneous maximum load factor, we benefit of the instantaneous maximum turning rate along the curve 6 which is a modification of the curve 5 with the constraint $n_{s}$ 
We have used the same two jet engines to plot the thrust limit in curves 3 and 4 . It now appears that with the high thrust engine, in curve 4 , although wc cannot hold the maximum rate at point $S_{1}$, steady turn at the local maximum rate at point $S_{2}$ using the corner Mach number at point $\mathrm{C}_{2}$ can be maintained.

\section{Optimum Turning}

It is proposed to change the heading by a certain angle $\Delta \psi=\psi_{\mathrm{f}}-\psi_{0}$, say of $180^{\circ}$, and at the same time bring the Mach number from $\mathrm{M}_{0}$ to $\mathrm{M}_{\mathrm{f}}$, in minimum time.

The dynamical system is governed by Eqs. (3), with the constraint (5). Since we do not specify the final position and by the fact that $\mathrm{x}$ and $\mathrm{y}$ are ignorable coordinates, we have the reduced system

$$
\begin{aligned}
& \sum_{d t}{ }_{a}^{g}(T-D) \\
& \frac{d \psi}{d t}=\frac{g}{a M} \tan \phi
\end{aligned}
$$

We have transformed the speed into the Mach number, in line with the dependence of the aerodynamic and engine characteristics on this parameter. The influence of the thrust angle-of-attack has been ignored at high speed range. The load factor is simply

$$
\mathrm{n}=\frac{\mathrm{L}}{\mathrm{W}}
$$

with the relation with the bank angle expressed as

$$
\mathrm{n}=\frac{1}{\cos \phi} \leq \mathrm{n}_{\mathrm{s}}
$$

We shall use the thrust magnitude and the load factor as the control for turning. Then with a parabolic drag polar as given in Ey. (32) we write the system (34) using the definition (35)

$$
\begin{aligned}
& \frac{\mathrm{dM}}{\mathrm{dt}}=\frac{\mathrm{g}}{\mathrm{a} W}\left[\eta \mathrm{T}_{\max }(\mathrm{M}, \mathrm{h})-\mathrm{q} S \mathrm{CDO}_{\mathrm{D}}(\mathrm{M})-\frac{\mathrm{n}^{2} \mathrm{~W}^{2} \mathrm{~K}(\mathrm{M})}{\mathrm{qS}}\right] \\
& \frac{\mathrm{d} \Psi}{\mathrm{dt}}= \pm \frac{\mathrm{g}}{\mathrm{a} M} \sqrt{\mathrm{n}^{2}-1}
\end{aligned}
$$

where

$$
q=\frac{1}{2} k p M 2
$$

As has been discussed in the previous section, the load factor used as the control is subject to the constraint

$$
1 \leq \mathrm{n} \leq \mathrm{n} *(\mathrm{M})=\min \left[\mathrm{n}_{\mathrm{s}}, \mathrm{n}_{\max }(\mathrm{M})\right]
$$

where

$$
n_{\max }(M)=\left(\frac{k p S}{2 W}\right) M^{2} C_{\text {Lmax }}(M)
$$

It is assumed that $a(h), p(h)$ are known as functions of the altitude, $\mathrm{C}_{\mathrm{D} 0}(\mathrm{M}), \mathrm{K}(\mathrm{M})$ and $\mathrm{C}_{\mathrm{L} \max }(\mathrm{M})$ are given as functions of the Mach number and that $\mathrm{T}_{\max }(\mathrm{M}, \mathrm{h})$ are prescribed in tabulated form for twojet engines. We notice that this is also applicable to other types of thrust producing engines such as the rocket or the turbofan.

It is proposed to bring the system from $\mathrm{M}_{0}, \psi_{0}=0^{\circ}$ to $\mathrm{M}_{\mathrm{f}}$ and $\psi_{\mathrm{f}}$ in minimum time, subject to the prescribed acrodynamic, thrust and load factor constraints.

For this minimum time problem, we introduce the adjoints $\mathrm{P}_{M}$ and $\mathrm{P}_{\psi}$ to form the I-Iamiltonian

$$
\begin{aligned}
H= & P_{M} \frac{g-g}{a W}\left[\eta T_{\max }-q S\left(C_{D 0}+\frac{n^{2} W^{2}-K}{q^{2} S^{2}}\right)\right] \\
& \pm P_{\psi} \frac{g}{a M} \sqrt{n^{2}-1}
\end{aligned}
$$

Concerning the thrust control, we consider the switching function $\mathrm{P}_{\mathrm{M}}$. Then in maximizing the Hamiltonian

$$
\begin{aligned}
& \text { If } \mathrm{P}_{M}>0 \text {, use } \eta=1 \text { for } \mathrm{T}_{\max } \\
& \text { If } \mathrm{P}_{M}<0 \text {, use } \eta=0 \text { for } \mathrm{T}=0 \\
& \text { If } \mathrm{P}_{M} \equiv 0 \text {, use intermediate } \eta \text { for } \mathrm{T}=\text { variable }
\end{aligned}
$$

The optimum trajectory is a sequence of subarcs from the boost, coast and sustained thrust control. For the load factor, or bank angle control, it is either at the maximum limit, or at an interior point such that $\partial \mathrm{H} / \partial \mathrm{n}=0$. Explicitly, we have

$$
\pm \sqrt{n^{2}-1}=\underset{4 W}{(K B)}\left(\frac{1 v_{1} r \psi}{K P_{M}}\right)
$$

It has been shown in Ref. 1 that while this interior load factor can be optimally used with the boost arc, the coast arc and the sustained arc are always conducted with $n$, or $\mathrm{n}_{\max }(\mathrm{M})$.

Therefore, the thrust and bank controls are governed by the adjoints $\mathrm{P}_{\mathrm{M}}$ and $\mathrm{P}, \quad$ Fkst for the adjoint $\mathrm{P}$, it is seen that it is a constant. 


$$
\mathrm{P}_{\psi}=\mathrm{C}_{1}
$$

On the other hand, it is not necessary to integrate the equation for $P_{M}$ since we have the Hamiltonian integral available

$$
\begin{aligned}
& P_{M} \underset{a W}{-g}-\left[\eta T_{\max }-q S\left(C_{D 0}+\frac{n^{2} W^{2}}{q^{2} S^{2}}\right)\right] \\
& \pm P_{\psi} \frac{g}{a M} \sqrt{n^{2}-1}=C_{0}
\end{aligned}
$$

By using the optimum relation (43) for the interior load factor, and setting $\pm \sqrt{n^{2}-1}=\tan \phi$, we have the equation

$$
\begin{aligned}
& \tan ^{2} \phi-2 c M \tan \phi+\left(\frac{\mathrm{kpS}}{2 \mathrm{~W}}\right) \frac{\mathrm{M}^{2}}{\mathrm{~K}}
\end{aligned}
$$

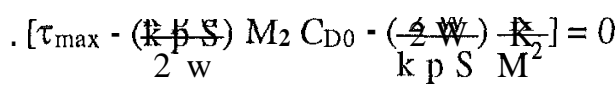

where

$$
\mathrm{c}=\frac{\mathrm{a} \mathrm{C}_{0}}{\mathrm{~g} \mathrm{P}_{\psi}}
$$

is an arbitrary constant to be selected so that the end conditions are identically satisfied. Of course $\tau_{\max }$ is the maximum thrust-to-weight ratio since this bank control implies that $\eta=1$.

It remains the case where the sustained arc at intermediate thrust is used. For this case we constantly have

$$
\mathrm{P}_{\mathrm{M}} \equiv 0
$$

for a finite time interval. Its time derivative also vanishes identically, and we have

$$
\frac{\mathrm{dP}_{\mathrm{M}}}{\mathrm{dt}}=-\left(\frac{\partial \mathrm{H}}{\partial \mathrm{M}}\right)_{\mathrm{P}_{\mathrm{M}=0}}= \pm \mathrm{P}_{\psi} \frac{\partial}{\partial \mathrm{M}}\left[\frac{\mathrm{g} \sqrt{\mathrm{n}^{2}-1}}{\mathrm{aM}}\right]=0
$$

It is not necessary to carry out the derivation, since by referring to Eq. (9), we see immediately that the condition (49) for sustained arc is the same as the condition for a local maximum of the tuining rate. The optimum Mach number is constant and the existence of the sustained arc depends on the magnitude of the thrust produced at the flight altitude. For example, in the case of Fig. 7 for turning at $14 \mathrm{kft}$, sustained arc is only possible at the corner Mach number with the high thrust engine.

\section{Computation of The Optimum Trajectory}

We consider the case of a 180 " turn at the altitude of $14 \mathrm{kft}$ with a starting Mach number $\mathrm{M}_{0}=1.2$. With the low thrust engine, there are no sustained arc and tlie optimum trajectory is a combination of boost arc (B) and coast arc (C). The switching occurs whenever $P_{M}=0$. From the Hamiltonian integral, we see that

$$
\frac{\mathrm{a}}{\mathrm{g}} \dot{\psi}=\frac{\sqrt{\mathrm{n}^{2}-1}}{\mathrm{M}}=\frac{\mathrm{a} C_{0}}{\mathrm{~g} \mathrm{C}_{1}}=\mathrm{c}
$$

Therefore, if more than one switching occurs, all the switchings are made at the same tuiiiing rate.

We have shown in Fig. 8 the time varying optimum turning rate leading to various final Mach numbers. The results are summarized in Table 1. The trajectories 1 and 2 are accelerating turn, with a B-arc and interior load factor. At tlie flight altitude, as specified by the dimensionless wing loading value $2 \mathrm{~W} / \mathrm{kpS}$, a value for the constant $\mathrm{c}$ is selected to calculate the optimum interior bank angle from Eq. (46). The equations of motion are integrated until $\psi_{\mathrm{f}}=180^{\circ}$ and the resulting value $\mathrm{M}_{\mathrm{f}}$ is checked with the prescribed value for iteration. The trajectories 3,4 , and 5 are decelerating turns. For the discussion, we refer to Fig. 7. For the trajectory 3 since $\mathrm{M}_{0}$ and $\mathrm{M}_{\mathrm{f}}$ are near of the corner Mach number where a local maximum for the tuining rate occurs, the trajectory starts with a C-arc to quickly move to near of the corner Mach number. But since the thrust for the example jet engine is not sufficiently high to maintain tlie constant peak turning rate, a switch to $\mathrm{B}$-arc has tlie sole purpose to hold the speed near of the optimum speed. Finally a switch to $\mathrm{C}$-arc near of tlie end is designed to match the prescribed Mach number. In this program, since the thrust control, either $\eta=0$ or $\eta=1$, is well defined and the boundary load factor, $n=n_{s}$ before the point $C_{2}$ and $n=n_{\max }(M)$ after the point, is known explicitly, the arbitrary constant c selected is used to iterate on the switching time to satisfy the end condition. We notice an interesting case when $\mathrm{Mr}_{\mathrm{r}}=0.8$, in the curve 4. In Fig. 7, by drawing a horizontal line slightly below the peak turning rate $S_{2}$, we see that this line intersect the curve for $\dot{\psi}$ in 3 points between $\mathrm{M}_{0}=1.2$ and $\mathrm{M}_{\mathrm{f}}=0.8$. We have adjusted this rate for matching and it results in 3 switches leading to the sequence C-B-C-B. The optimum control strategy is clear. Since it is a decelerating trajectory and a peak turning rate is at $\mathrm{C}_{2}$, the decelerating trajectory starts with a $\mathrm{C}$-arc, with the instantaneous maximized turning rate increasing with the time. In this duration $\mathrm{P}_{\mathrm{M}}<0$. When $\mathrm{P}_{\mathrm{M}}=0$, we reach the turning rate for switching which is $\dot{\psi}_{\mathrm{s}}=\mathrm{cg} / \mathrm{a}<\dot{\psi}_{\max }$. The thrust control changes to $\mathrm{T}_{\max }$ while $\dot{\psi}$ continues to increase, passes through $\dot{\psi}_{\max }$ and returns to $\dot{\psi}_{\mathrm{s}}$ where the B-arc changes to 
C-arc. After this switch $\dot{\psi}$ tends to its minimum before increasing again and a $\mathrm{C}$-are is appropriate to speed up the passage through the period of low turning rate. After passing through its minimum, $\dot{\psi}$ increases and when it attains $\dot{\psi}_{\mathrm{s}}$ tlie control changes to 13 -arcuntil $\mathrm{M}_{\mathrm{f}}$.

We should realize that since tlie constants $\mathrm{C}_{0}$ and $C_{1}$ are the same throughout the entire trajectory from $t_{0}$ to $\mathbf{t}_{\mathrm{f}}$, the constant $\mathrm{c}$ is always the same. Therefore, while on a $\mathrm{B}$-arc, there is always a possibility of interior load factor as given by Eq. (46), which can be constantly calculated and compared with $n_{*}(\mathrm{M})$, once a value of $\mathrm{c}$ has been selected. Finally, for the case where $M_{f}=0.6$, we have the $C-B$ sequence as shown in trajectory 5 . This is because the solution for c gives a high turning rate near of the point $S_{1}$ at switching time. A C-arc is used to quickly bring the system to the condition of high turning rate followed by a $\mathrm{B}$-arc to keep the system near of this absolute optimum rate.

Table 1. Types of trajectory with various final Mach numbers. Case of $\mathrm{M}_{0}=1.2, \psi_{\mathrm{f}}=180^{\circ}, \mathrm{h}=14 \mathrm{kft}$

\begin{tabular}{|c|c|c|c|c|}
\hline $\mathrm{N}_{0}$ & $\mathrm{M}_{\mathrm{f}}$ & $\mathrm{C}$ & $\mathrm{t}_{\mathrm{f}}(\mathrm{sec})$ & Type \\
\hline 1 & 2.0 & 5.5349 & 65.63 & $\mathrm{~B}$ \\
2 & 1.6 & 4.4242 & 43.54 & $\mathrm{~B}$ \\
3 & 0.9 & 4.9305 & 20.82 & C-B-C \\
4 & 0.8 & 5.1381 & 20.05 & C-B-C-B \\
5 & 0.6 & 7.9519 & 18.13 & C-B \\
\hline
\end{tabular}

In the trajectories in Table 1, we have used the example jet engine in Ref. 4 and the thrust is not sufficiently high to keep the maximum rate $\dot{\psi}_{\max }$ at the coiner Mach number, From Fig. 7, with a higher thrust limit passing above the point $\mathrm{C}_{2}$, as shown in the curve 4 , we have recalculated the trajectory leading to $\mathrm{M}_{\mathrm{f}}=0.9$, and we have the type $\mathrm{C}-\mathrm{S}-\mathrm{C}$ with a slightly better time of $\mathrm{t}_{\mathrm{f}}=20.489$ seconds. For this trajectory, a C-arc is used to quickly reduce the Mach number to the corner Mach number for maximum tuining rate $\dot{\psi}_{\max }$. The load factor used is $n=n_{s}$ and the heading change is $\Delta \psi_{1}$. The maximum thrust is then reduced to maintain this constant rate for a heading change $\Delta \psi_{s}$. Finally a $\mathrm{C}$-arc is used with $\mathrm{n}_{\max }(\mathrm{M})$ to decrease the Mach number to $\mathrm{M}_{\mathrm{f}}$ with a heading change of $\Delta \psi_{2}$. Since $\Delta \psi_{1}$ and $\Delta \psi_{2}$ are well defined, the time on the intermediate (or singular) $\mathrm{S}$-arc is the time necessary to make up the change $\Delta \psi_{\mathrm{s}}$ for a total of $180 "$ heading change.

\section{Conclusions}

As a contribution to research literature in modern flight dynamics ${ }^{5-10}$, in this paper tlie turning performance of modem fighter aircraft has been examined. In general, in the exact formulation of the dynamical system, the thrust vector has a small component Tsin $\alpha$ in the direction of the lift force and the optimum angle-of-attack for maximum turning rate and the one for minimum turning radius are always larger than the stall angle-of-attack where the liftcoefficient reaches a maximum and starts to decrease drastically. With very high thrust, the instantaneous maximum turning rate, and the instantaneous minimum tuining radius are achieved at the maximum possible angle-of-attack.

In optimum turning in a horizontal plane, in which the prescribed end-points Mach numbers are high for operational purpose, the strategy for minimum time maneuvers is to hold the turning rate near of the local maximum rate. For supersonic aircraft, there may exist two peak turning rates at a certain range of altitude. With low or moderate maximum thrust output, the optimum control leads to switching between boost arcs and coast arcs such that the end-conditions are satisfied. Trajectories with one, two and even three switchings have been calculated. With a high thrust engine, the optimum control usually leads to inserting an intermediate thrust arc to keep maximum constant turning rate in the middle portion of the turn. The formulation allows the consideration of a variety of thrust producing propulsion systems which include the rocket, the turbojet and the turbofan.

\section{Appendix}

In plotting Figs. 3 - 5, we have used the functions $\mathrm{C}_{\mathrm{D}}(\alpha)$ and $\mathrm{C}_{\mathrm{L}}(\alpha)$ based on data points from Ref. 3 and extended to $\alpha=70^{\circ}$. In the computation of the optimum maneuvers for Figs. 6 - 8, we have used tlie aerodynamic data $\mathrm{C}_{\mathrm{D} 0}(\mathrm{M})$ and $\mathrm{K}(\mathrm{M})$ and the maximum thrust $\mathrm{T}_{\max }(\mathrm{M}, \mathrm{h})$ for the supersonic fighter considered in Ref. 4 with $\mathrm{W}=38000 \mathrm{lbs}, \mathrm{S}=500 \mathrm{ft}^{2}$. The limitation for the stall lift coefficient $C_{\text {max }}(M)$ is based on the smooth spline approximation from the data point

\begin{tabular}{l|lllll}
$\mathrm{M}$ & 0 & 0.6 & 0.7 & 0.9 & 1.0 \\
\hline $\mathrm{C}_{\mathrm{Lmax}}$ & 0.95 & 0.95 & 0.8344 & 0.3875 & 0.329
\end{tabular}




\begin{tabular}{l|llll}
$\mathrm{M}$ & 1.2 & 1.6 & 2.4 & 3.2 \\
\hline $\mathrm{C}_{\mathrm{Lmax}}$ & 0.34 & 0.45 & 0.3617 & 0.34
\end{tabular}

The example engine thrust is low as displayed in curves 3 in Figs. 6 and 7.

To include the sustained arc we have magnified this maximum thrust by the factor $\mathrm{f}(\mathrm{M})$ which gives an average increase of about $35 \%$. This leads to a larger thrust limit as shown in curves 4 in Figs. 6 and 7.

\section{Acknowledgment}

Part of this work was supported by the R.O.C. Aeronautical Industry Development Center.

\section{$\underline{\text { References }}$}

1.N.X. Vinh, Ontimal Traiectories in Atmospheric Flight, Elsevier Scientific Publishing Company, Amsterdam, 1981.

2. N.X. Vinh, Flight Mechanics of Hish-Performance Aircraft, Cambridge University Press, England, 1993.

3. Lamar, J.E., and Frink, N.T., "Aerodynamic Features of Designed Strake-Wing Configurations," Journal of Aircraft, Vol. 19, No. 8, 1982, pp. 639-646.

4. Bryson, A. E., Desai, M. N., and Hoffman, W. C., " Energy-State Approximation in Performance Optimization of Supersonic Aircraft," Journal Of Aircraft, Vol. 6, No. 6, 1969, pp. 481-488.

5. Hedrick, J. K., and Bryson, A. E., "Three-Dimensional, Minimum-Time Turns for a Supersonic Aircraft," Journal of Aircraft, Vol. 9, No. 2, 1972, pp. 115-120.

6. Hedrick, J. K., and Bryson, A. E., "Three-Dimensional, Minimum Fuel Turns for a Supersonic Aircraft," Journal of Aircraft, Vol. 9, No. 3, 1972, pp. 223-229.

7. Hedrick, J. K., and Bryson, A. E., "Minimum Time Turns for a Supersonic Airplane at Constant Altitude," Journal of Aircraft, Vol. 8, No. 3, 1971,pp. 182-187.

8. Bilimoria, K.D., Cliff, E. M., and Kelley, H. J., "Classical and Neo-Classical Cruise-Dash Optimization," Journal of Aircraft, Vol. 22, No. 7, 1985, pp. 555-560.

9. Chichka, D. E., Sliankar, U. J., Cliff, E. M., and Kelly, H. J., " Cruise-Dash-Climb Analysis of Airbreathing Missile," Journal of Guidance, Control, and Dynamics, Vol. 11,No. 4, 1988, pp. 293-299.

10. Bocvorov, S., Lutze, F. H., and Cliff, E. M., " Time Optimal Reorientation Maneuvers for a Combat Aircraft," Journal of Guidance, Control, and Dynamics, Vol. 16, No. 2, 1993, pp. 232-240.

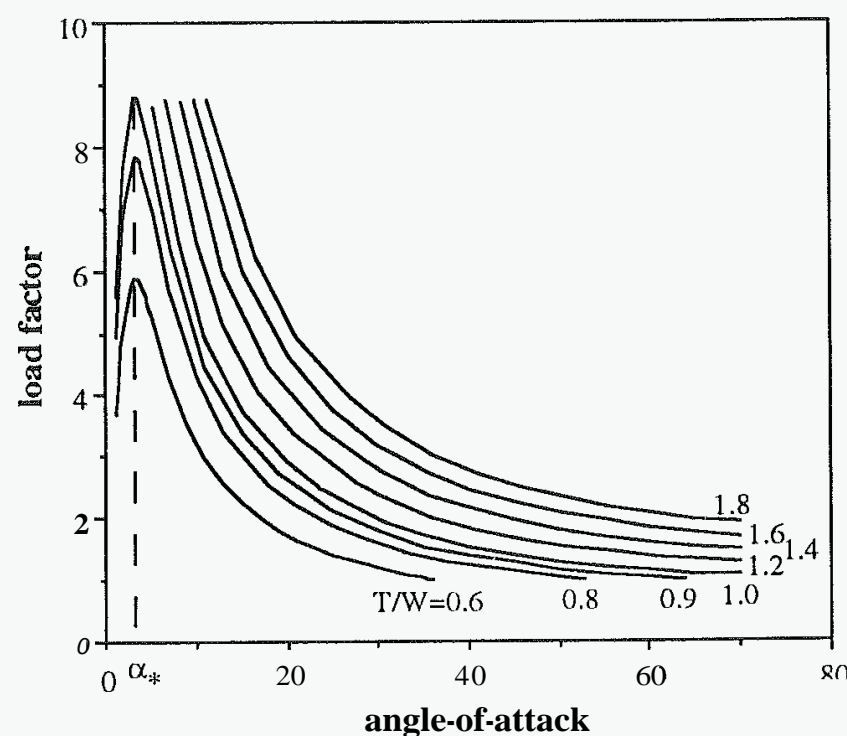

Fig. 3 Load factor as function of the angle-ofattack

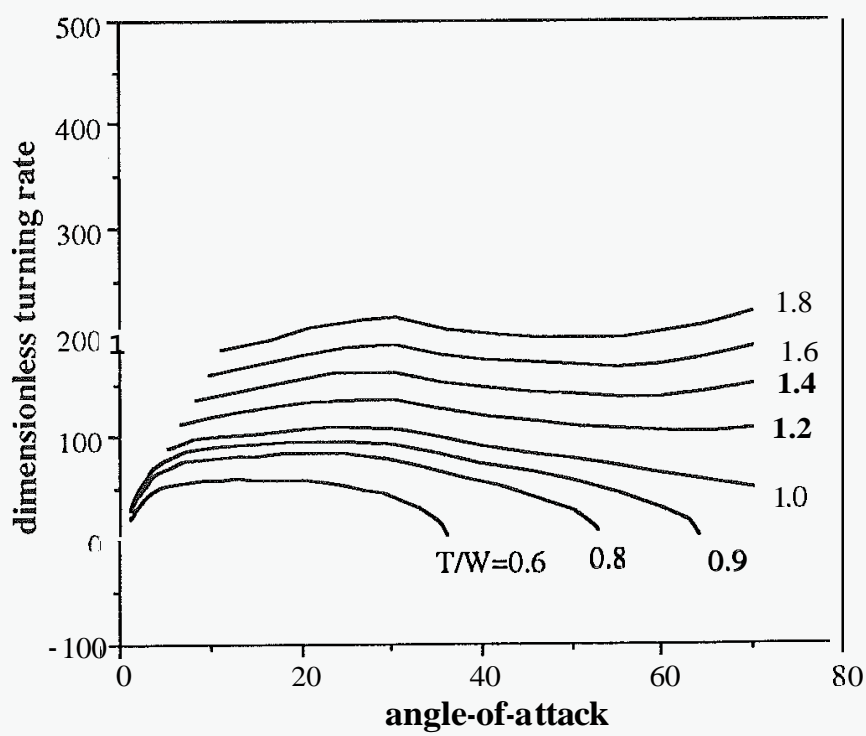

Fig. 4 Dimensionless turning rate as function of the angle-of-attack 


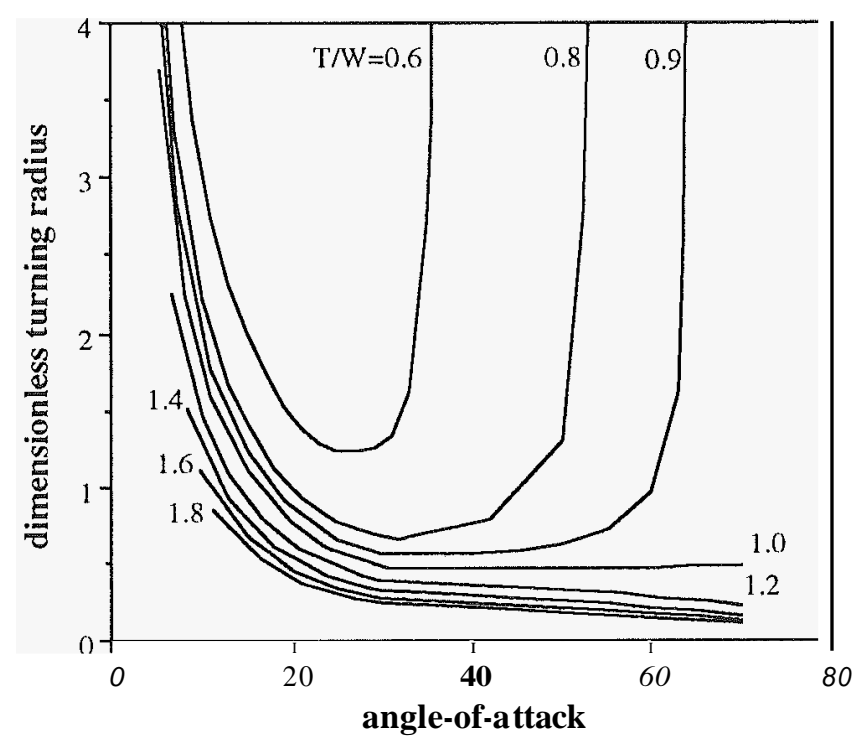

Fig. 5 Dimensionless turning radius as function of the angle-of-attack

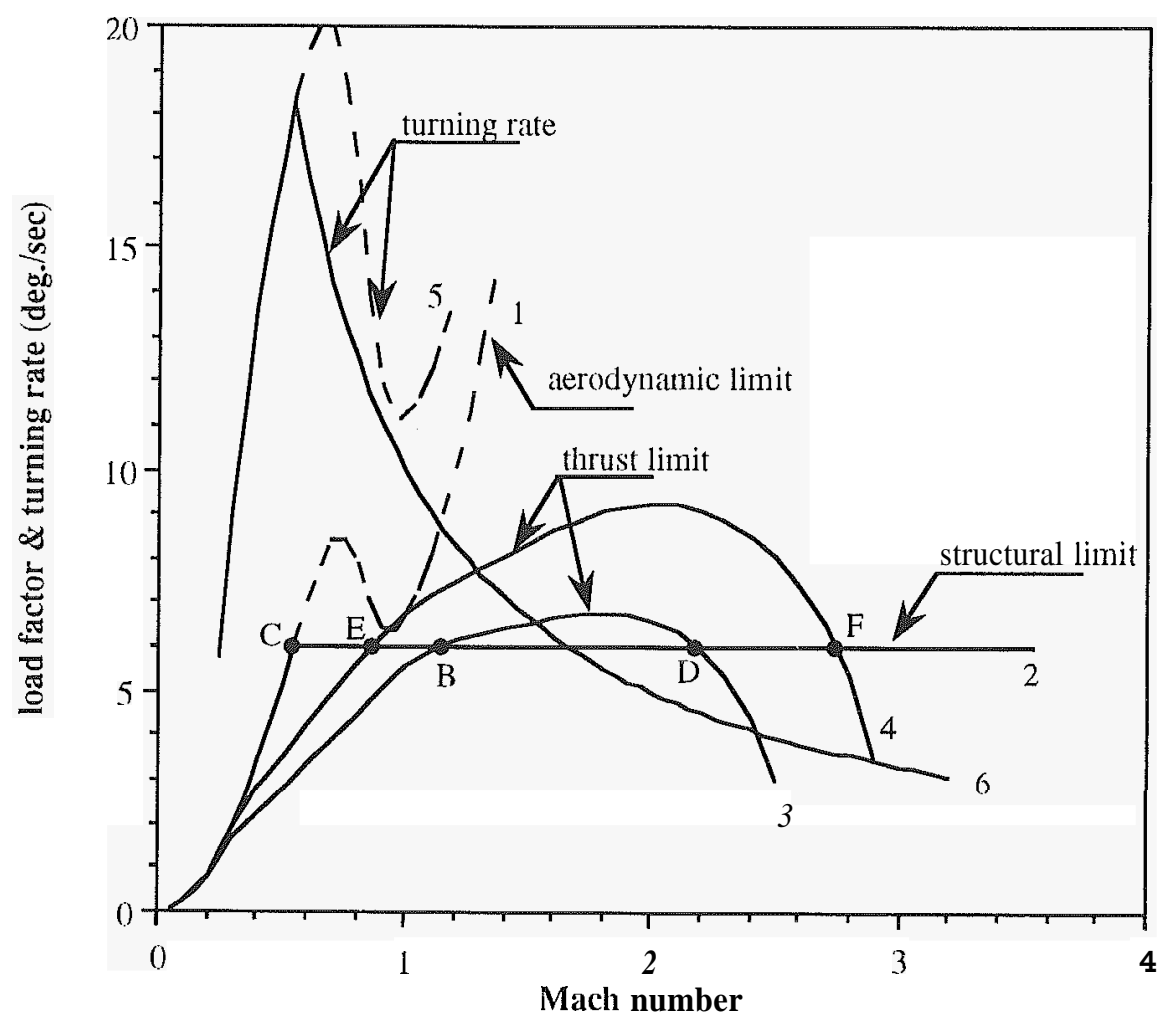

Fig. 6 Domain of maneuverability and maximum turning rate at 5,000 $\mathrm{ft}$ 


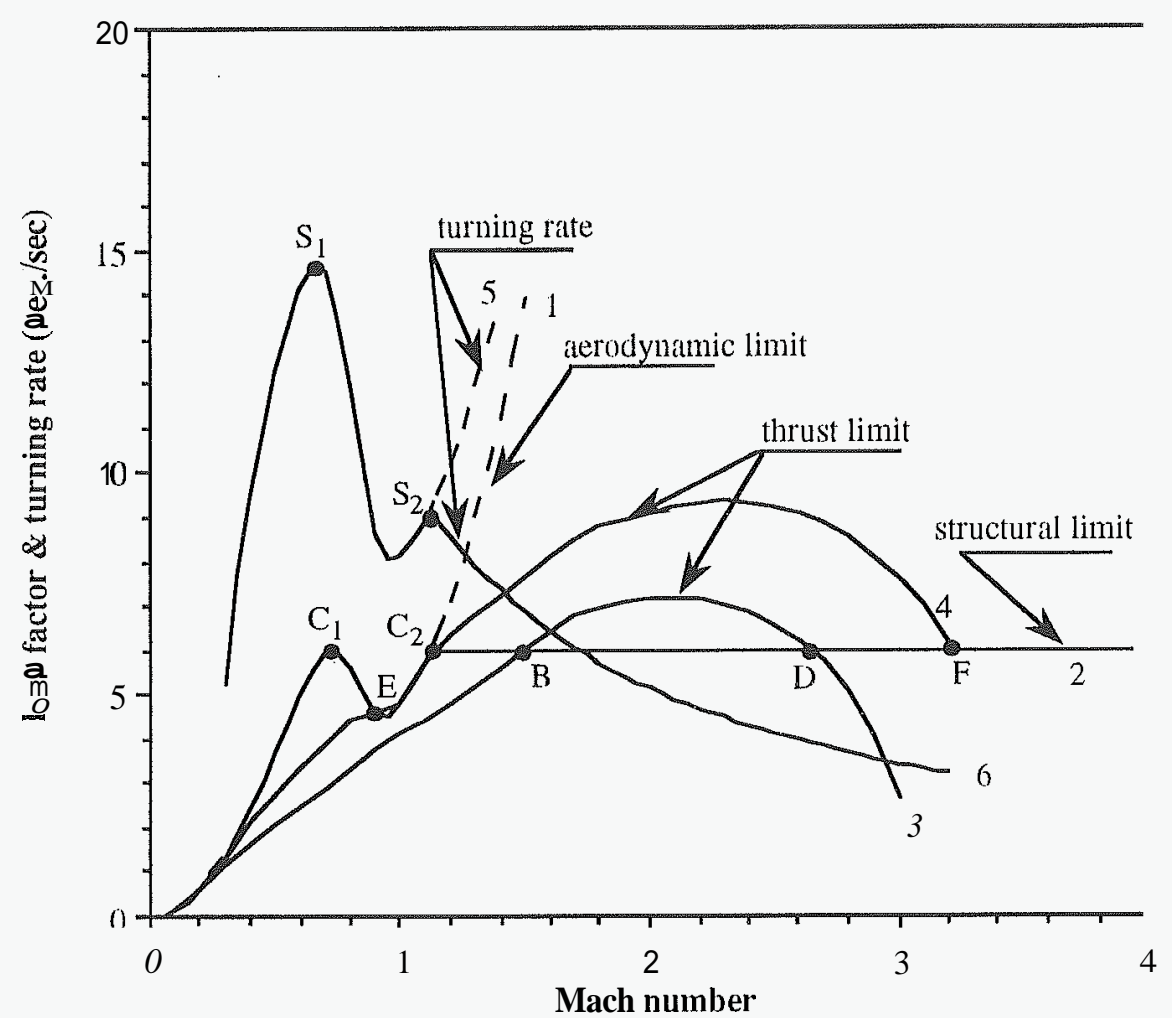

Fig. 7 Domain of maneuverability and maximum turning rate at $14,000 \mathrm{ft}$

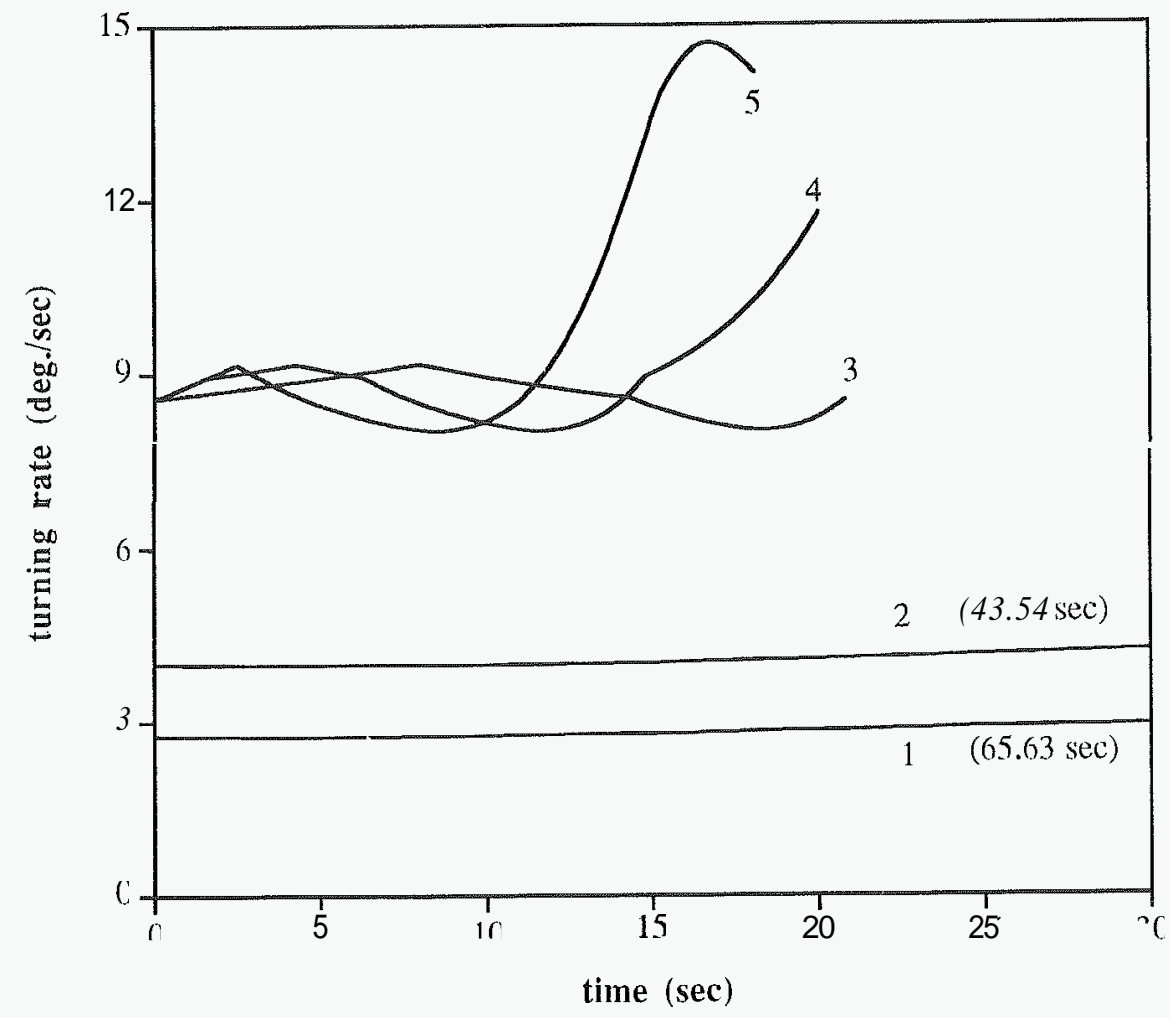

Fig. 8 Variation of the optimum turning rate for a 180 " turn at $\mathrm{h}=14 \mathrm{kft}$ 